\title{
Supporting Remote Therapeutic Interventions with Mobile Processes
}

\author{
Marc Schickler, Rüdiger Pryss, Johannes Schobel, Manfred Reichert \\ Institute of Databases and Information Systems, Ulm University, Germany \\ \{marc.schickler, ruediger.pryss, johannes.schobel, manfred.reichert\}@uni-ulm.de
}

\begin{abstract}
Many studies have revealed that homework (e.g., relaxation exercises) are crucial for remote therapeutic interventions. In this context, to monitor whether patients actually perform their homework and to check whether they perform it in the right way constitute complex tasks. So far, therapeutic interventions have not been properly supported by IT systems and, hence, the opportunities provided by mobile assistance have been neglected. For example, a smart mobile device may notify a patient about an assigned homework or motivate him to accomplish it in time. Moreover, the patient might be further assisted through a video providing detailed instructions. In turn, the smart mobile device could inform the therapist of the homework outcome. In practice, a proper support of the various types of homework is challenging, even when using modern IT systems. To remedy this drawback, we propose an approach integrating mobile services with process management technology in order to enable the complex coordination tasks that become necessary in connection with homework. For example, a process might enable remote monitoring of homework, giving therapists the opportunity of timely adjustments. In addition, the approach allows involving researchers by providing them with valuable data (e.g., heart rate) gathered during and after homework. This paper presents an approach for creating processes that run on smart mobile devices and enable flexible remote therapeutic intervention support. Such mobile approach significantly enhances therapy assistance on one hand and mobile homework-related scenarios on the other.
\end{abstract}

Keywords-Mobile service, therapeutic intervention, mobile context, mobile process, mobile assistance, mobile therapy

\section{INTRODUCTION}

According to numerous clinical studies, therapeutic interventions constitute a fundamental part of most therapies to increase their efficacy [1], [2]. The variety of therapeutic interventions, in turn, ranges from simple medication intakes up to complex homework, which are assigned by a therapist to the patient. Taking the diversity of useful homework into account, therapists crave for a proper IT support. In line with this trend, recent studies (e.g., [3]) revealed that the use of smart mobile devices is particularly demanded by therapists [4]. Unfortunately, existing mobile approaches still show drawbacks. First, they mostly provide specifically tailored mobile applications and, hence, do not deal with the diversity of therapeutic interventions. Second, they focus on the therapist-patient relationship, neglecting other promising opportunities. Third, features provided by current smart mobile devices (e.g., sensors) have not been broadly used for therapeutic interventions yet.

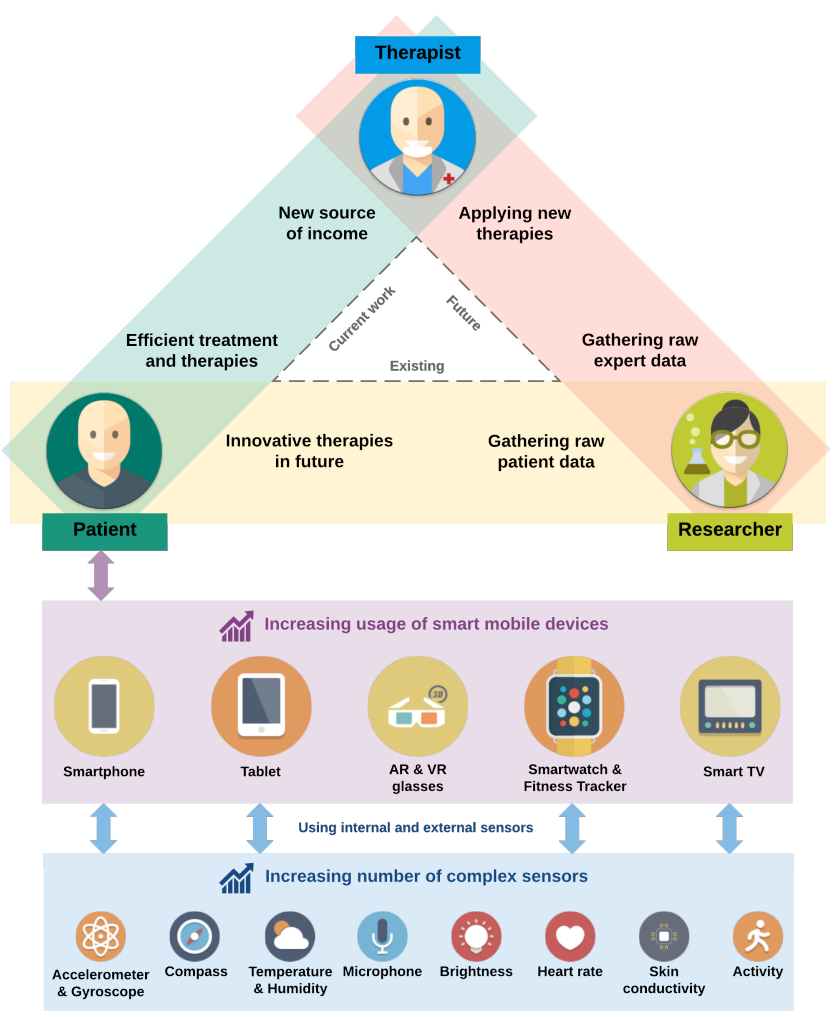

Figure 1: Pursued Objectives

Considering these drawbacks, Fig. 1 illustrates three fundamental objectives relevant for a proper therapeutic intervention support. First of all, in addition to smartphones and tablets, emerging technologies like smartwatches need to be integrated to address current demands of mobile users. Second, the opportunities provided by smart mobile device sensors should be utilized as well. Third, the therapistpatient relationship needs to be extended to a therapistpatient-researcher relationship to take benefit from the valuable data collected with the smart mobile devices over time. If these objectives are met, the following advantages will result:

1) Patients will obtain better treatment as therapeutic interventions may be personalized, i.e., adjusted to the specific situation of the patient at hand.

2) Therapists are enabled to provide an optimized and more efficient treatment as they can monitor and 
dynamically adjust therapeutic interventions remotely. Respective adjustments, for example, might foster innovative billing models.

3) In the longer term, patients might benefit from new therapies developed on the basis of the data collected.

4) Therapists can use emerging therapies discovered by researchers based on the data gathered over time.

5) Researchers discover new therapies or gain new insights into diseases based on the data provided by patients (e.g., sensor data) as well as by therapists (e.g., context-based therapy results).

As illustrated, the IT support of therapeutic interventions is complex. In particular, all (remote) issues not taking place in the scope of personal meetings (e.g., homework) between therapist and patients are technically challenging. On the other, these remote issues require a tight integration with issues (e.g., general treatment adaptations) that occur during the personal meetings. Consequently, a holistic view on the entire procedure is crucial. From a technical perspective, an overall protocol must be developed to coordinate all required issues. In the given context, the development of such a protocol is particularly challenging as both stationary as well as mobile systems must be considered. Therefore, we advocate an approach based on mobile processes to enable the required coordination protocol. If the latter can be established, the efficacy of therapeutic interventions may be increased since an effective monitoring of the aforementioned remote issues becomes possible. Based on the resulting monitoring data, for example, required adaptations to the therapeutic intervention procedure can be realized in real time as well as in a more patient-centric manner.

To realize this protocol, first of all, we defined a metamodel taking the complex organization of therapeutic interventions into account. Based on the meta-model, we developed a technical solution executing processes on smart mobile devices. These mobile processes enable us to perform complex therapeutic interventions for patients assisted by their smart mobile devices.

This paper shows how mobile processes are realized and in what way they effectively support therapeutic interventions. In detail, fundamental requirements on one hand, and the meta-model, main concept, and proof-of-concept prototype are presented on the other. The remainder of the paper is structured as follows: Section II discusses elicitated requirements. Section III presents the meta-model derived from these requirements. In Section IV, the support of mobile processes is discussed, whereas Section V deals with relevant sub-process patterns in more detail. Section VII gives insights into the proof-of-concept prototype. Finally, Section VIII discusses related work and Section IX concludes the paper with a summary and outlook.

\section{REQUIREMENTS}

First of all, we summarize how therapeutic interventions are traditionally accomplished (i.e., without IT support): The therapy starts with a first personal meeting of the therapist and the patient. During this meeting as well as in follow-up sessions, the therapist creates a comprehensive anamnesis for the case conceptualization, upon which the remote therapeutic interventions (e.g., homework) are planned. Based on the anamnesis, the therapist compiles a therapy plan, consisting of (1) therapeutic interventions, (2) personal appointments with the patient, and (3) the duration required to achieve a positive therapy effect for the patient. Concerning the therapeutic interventions, two intervention types need to be created by therapists, the one applied during the personal meetings and the one performed by the patients at home between the meetings with the therapist. As one of the most crucial drawbacks of this traditional procedure, homework cannot be remotely monitored and, hence, therapists are unable to timely adjust ongoing homework. Additionally, the exchange of feedback between therapist and patients is not efficiently supported. As the mobile processes we target at must cover the variety of therapeutic interventions, profound insights into real-world scenarios are indispensable. In general, respective insights should be gathered in the context of large-scale case studies over longer period of times to better understand actual requirements. Ourselves, we base the development of the mobile process support on the experiences we gathered when realizing complex mobile applications that support therapeutic interventions in the large scale [5]-[7].

In these case studies, therapists were provided with specifically tailored mobile applications. Thereby, all studies revealed that, when using mobile applications, therapeutic interventions can be supported more efficiently [6]. Regarding the case study in a psychosomatic in-patient clinic, for example, we learned that the support of homework with a mobile application is considered as useful in almost all patient cases [6]. According to a particular feature demanded in this context, patients shall be notified on their smart mobile devices about the need to perform their homework. The latter, in turn, shall be scheduled by a therapist to allow for a better therapy effect. Table I summarizes the elaborated requirements that need to be met by mobile processes in order to efficiently support therapeutic interventions. We divide the requirements into three categories. (1) Requirements related to homework. For example, therapists should be able to assign media elements to homework, which are then presented to the patient on his smart mobile device. (2) Requirements related to the context of a homework. This context enables us to cope with the demands of therapists on one hand (e.g., performance of a homework after getting up) and enables researchers to gather context-sensitive data on the other. (3) Requirements related to smart mobile devices. 


\begin{tabular}{lll}
\hline No & Title & Description \\
\hline \multicolumn{3}{c}{ Category I: Homework } \\
1 & Notification & Create notification schema for homework \\
2 & Configuration & Configure homework (e.g., context, and sensors) \\
3 & Versioning & Create and manage homework versions \\
4 & Feedback & Configure feedback schema for homework \\
5 & Reuse & Use homework for different scenarios \\
6 & Media & Use media elements for homework (e.g., videos) \\
\hline Category II: Context \\
7 & Notification & Bind notification schema to context \\
8 & Notification & Provide advanced notifications \\
9 & Configuration & Configure context (e.g., location, etc.) \\
10 & Versioning & Create and manage context versions \\
\hline Category III: Smart Mobile Device \\
11 & Notification & Apply notification schema \\
12 & ACR & Evaluate context \\
13 & Sensing & Use smart mobile device sensors \\
14 & Media & Provide media elements \\
\hline \multicolumn{3}{c}{$A C R$} \\
\hline \multicolumn{3}{c}{$A$ Automatic Context Recognition } \\
\hline
\end{tabular}

Table I: Elicitated Requirements

For example, the smart mobile device shall provide media elements to assist patients in performing their homework. Altogether, 14 fundamental requirements were elicitated.

\section{META-MODEL}

First of all, the definition of the meta-model needs to incorporate the lessons learned from the real-world projects. Second, it needs to capture the complexity of therapeutic interventions consisting of comprehensive homework, e.g., daily back exercises. Homework, in turn, comprise several activities. For example, the following activities may have to be performed in the context of a daily back exercise: warm up, strengthening, and cool down. Therefore, the meta-model considers homework as well as activities as core entity types. Furthermore, the meta-model considers the context (e.g., being in the living room), in which the homework shall be performed as well as it takes the sensors (e.g., heart rate) of smart mobile devices into account.

Accordingly, the meta-model is built up on three levels (cf. Fig. 2). On the first level, a Therapeutic Intervention consists of 1 up to $n$ Homework, for which an execution order needs to be defined. On the second level, a particular Homework encompasses a Notification, an Exercise, and a Feedback. In addition, the meta-model considers a Context and a Sensor on the second level. Sensor covers, for example, the sensors of smart mobile devices gathering data during an exercise (e.g., heart rate). While a Sensor is related to an Exercise and a Context, a Context may be related to a Notification and an Exercise. On the third level, the highlighted entities encompass activities, which either need to be performed by a patient (e.g., a warm up activity after getting up) or be automated (e.g., sensing the heart rate during a warm up), i.e., run on smart mobile device of a patient.

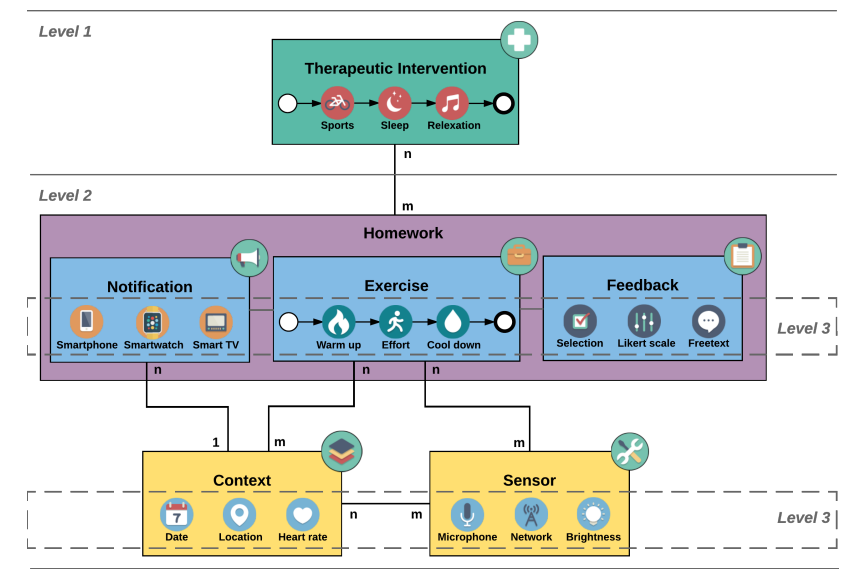

Figure 2: Meta-Model

\section{Mobile Process}

This section introduces the mobile processes developed on the basis of the meta-model. Fig. 3 shows a therapeutic intervention represented in terms of a mobile process. To be more precise, the mobile process consists of three process fragments. Each fragment, in turn, corresponds to a particular homework, whose execution order is determined by the mobile process. Homework, in turn, always encompasses three mobile process activities, i.e., a notification activity, an exercise activity, and a feedback activity. These activities, in turn, are always executed in the order Notification $\rightarrow$ Exercise $\rightarrow$ Feedback. That means, a patient is notified that an exercise needs to be accomplished. In turn, the patient shall provide feedback to the therapist. In practice, each activity is executed on a smart mobile device. Finally, homework are always executed in a particular context. For example, a homework might have to be performed in the living room after getting up. Note that the concrete implementation of all activities representing homework and being executed on the smart mobile device depends on the concrete homework scenario on one hand and on the used mobile platform on the other.

The analysis of the real-world scenarios revealed that activities representing exercises require a further itemization (cf. Fig. 4, Exercise level). To be more precise, therapists want to create exercises composed on several activities. For example, an exercise may comprise a warm-up activity, a push-up activity, and a cool-down activity. In addition, therapists may want to use the sensors of smart mobile devices to collect patient data during these activities. We therefore integrated a context-based sensing activity with exercises. Note that our practical insights revealed that both the notification and the feedback activity must be itemized in the same way as the exercise activity. Since the notification and feedback activities are similar to the exercise activity, we omit a more detailed discussion.

Practical insights further showed that exercise activities 


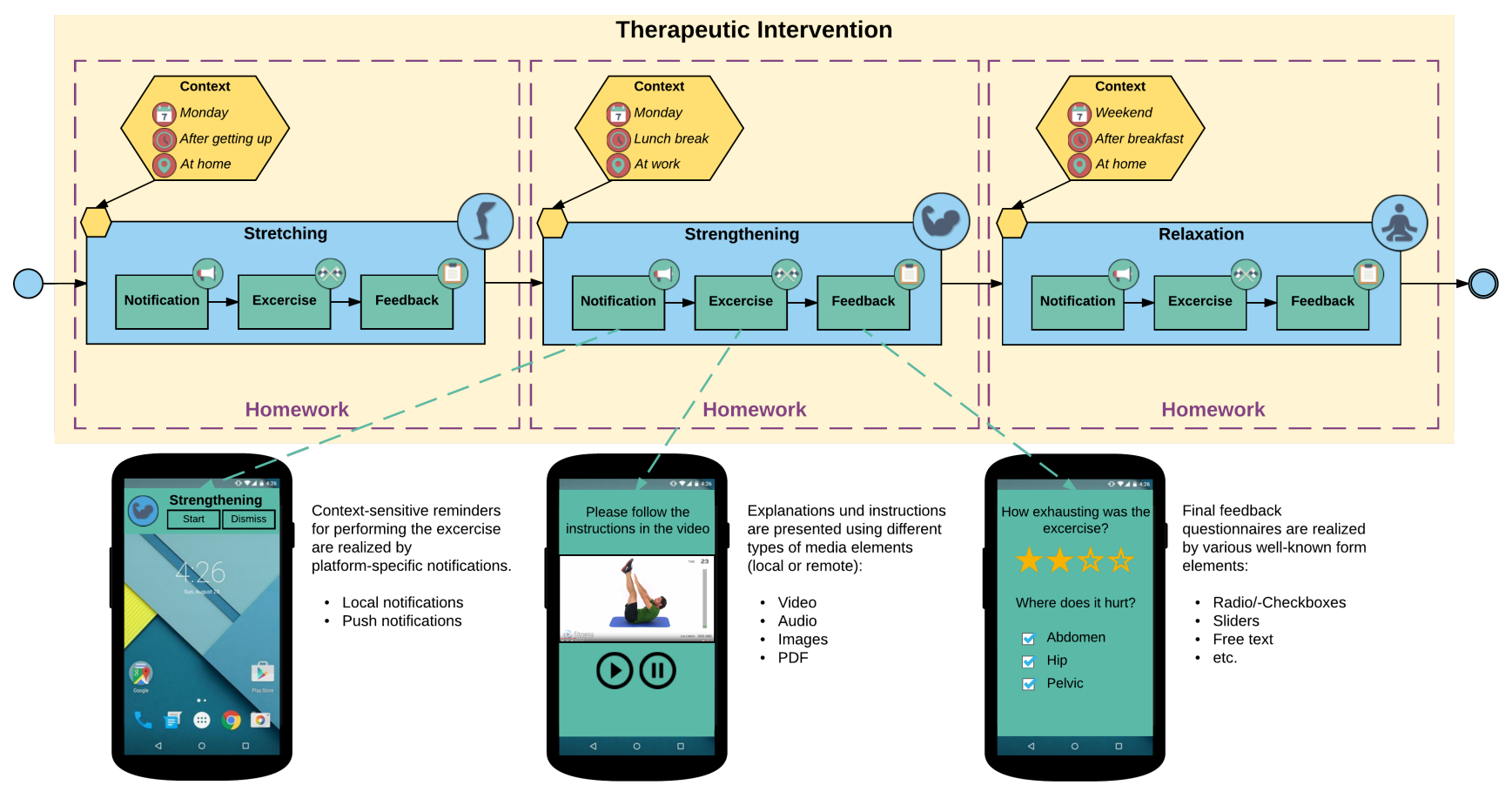

Figure 3: Remote Therapeutic Intervention with three Homework Fragments

require a more flexible concept to meet the requirements of therapists. Therefore, we allow for the implementation of exercise activities as sub-processes. For example, activity effort may be implemented as a sub-process (cf. Fig. 4, Subprocess level), containing activity swimming, followed by a decision that evaluates whether swimming shall be followed by activity biking or activity running. If activity biking is chosen, the biking speed will be measured by a contextbased sensing activity. Note that the contextual information, required to evaluate whether biking or running shall be chosen, needs to be specified by therapists.

Fig. 4 illustrates the described exercise itemization as well as the possible use of sub-processes. Furthermore, it is shown that the mobile processes are organized in five different levels. These levels constitute the basis for the modeling component that enables therapists to create therapeutic interventions themselves. More precisely, the modeling component offers features that consider the different levels in order to guide the therapists during the creation of therapeutic interventions in terms of mobile processes.

Regarding guidance, therapists are restricted to only use elements that are allowed for a specific level. In particular, on the exercise level, our modeling component provides various activity templates. The latter cover frequent scenarios occurring in the context of exercises. For example, the modeling component provides activity templates for measuring the heart rate or filling in a questionnaire. Note that therapists may create exercises solely based on the available activity templates. In practice, however, there exist scenarios in which the provided activity templates are not sufficient. For example, therapists may request an activity template for measuring skin conductance, while filling in a questionnaire in parallel. In such a case, the application
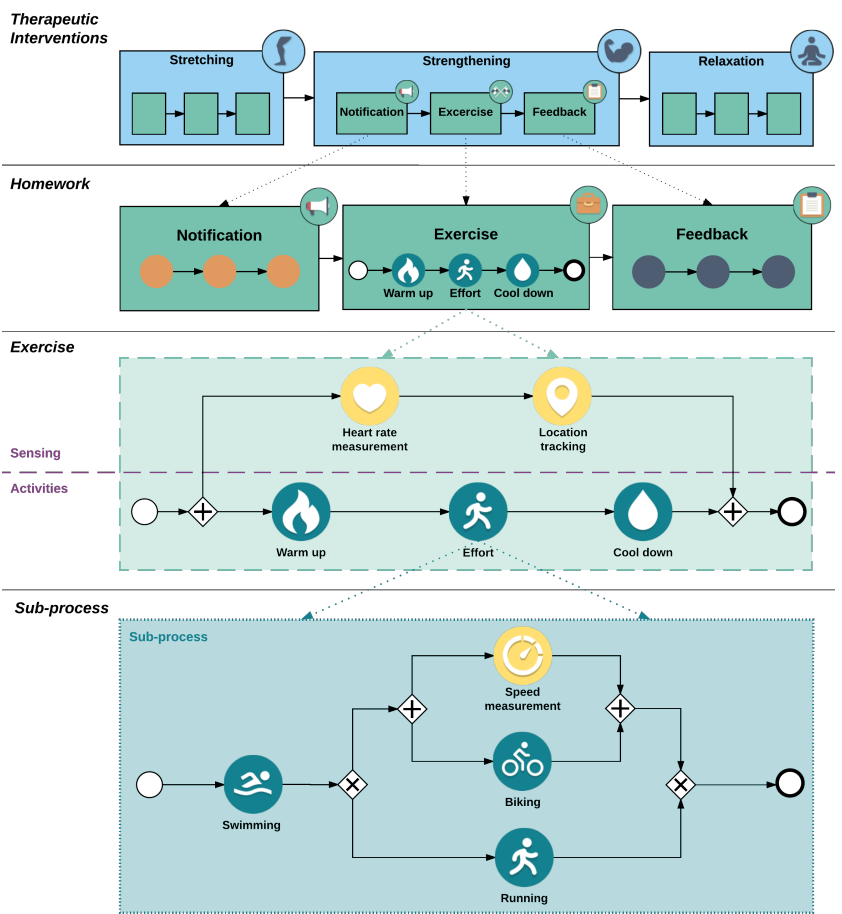

Figure 4: Mobile Process 
developer needs to implement the missing activity template. After releasing the newly implemented activity template, it may be used by therapists when modeling the exercise.

Therapeutic interventions created with the modeling component are transformed into executable processes running on smart mobile devices. The corresponding transformation procedure ensures that the resulting mobile processes can be correctly executed by a mobile process engine (e.g., the one we presented in [8]). Finally, the mobile process is deployed to the mobile application, which relies on the mobile process engine for executing the mobile processes.

\section{Sub-PRocess PATtERnS}

If therapists request new activity templates for defining concrete therapeutic interventions, these templates must be realized by an application developer. In several case studies, we learned that the development of new activity templates is (1) easily possible and (2) can be mostly accomplished without involving therapists [9]. Regarding the use of subprocesses, we further learned that requests from therapists frequently require the involvement of application developers, causing challenging debates between application developers and therapists. For example, two activities may have to be executed concurrently. However, contemporary mobile process engines do not provide such concurrency support. For this purpose, a specifically implemented sub-process is required, which can then be executed by the mobile process engine.

Regarding the support of application developers, we identified four fundamental patterns, which significantly ease the handling of sub-processes for them. We introduce these subprocess patterns in the following and discuss how they are evaluated during the development.

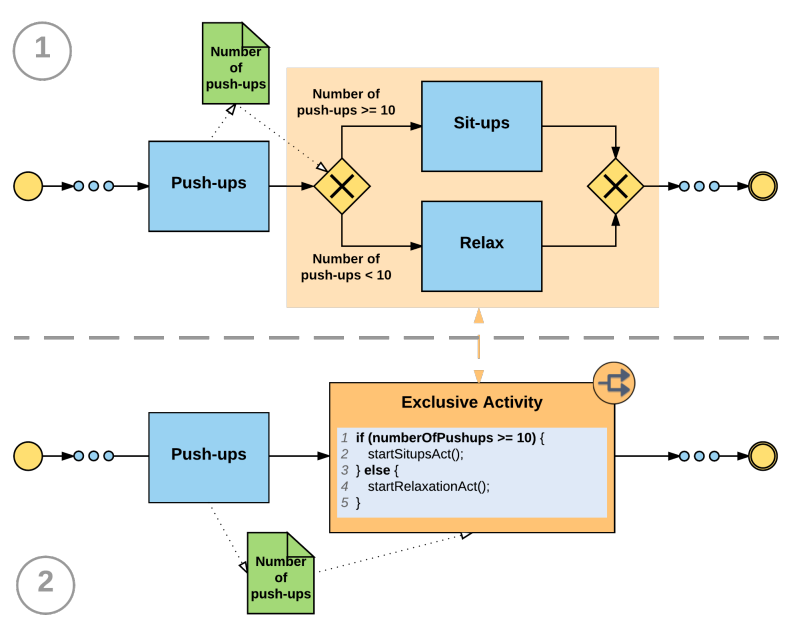

Figure 5: Decision Pattern

\section{A. Decision Pattern}

Fig. 5(1) shows a sub-process pattern, called Decision Pattern, in which two activities may be chosen alternatively (i.e., exclusive decision); i.e., during run time exactly one of the two activities may be executed. The decision, which activity will be actually chosen, is based on data that is (1) produced by a preceding activity or (2) provided by the therapist. In Fig. 5(1), for example, depending on the number of performed push-ups and determined by the pushups, the mobile process engine decides at run time whether the patient shall perform sit-ups or relax.

When a decision as shown in Fig. 5(1) is required by a therapist, an application developer must particularly evaluate if one (e.g., sit-ups in Fig. 5(1)) or more activity templates not exist. In the latter case, the application developer must decide whether to provide the missing activity templates or to replace the entire Decision Pattern with a new activity template instead of the pattern. To be more precise, an application developer is supported in deciding whether he applies the Decision Pattern or uses a specifically implemented activity template. For example, if complex forms are required for missing activity templates of the Decision Pattern, in most cases, a new activity template replacing the entire Decision Pattern fits practical demands best.

\section{B. Parallel Pattern I}

The approach of replacing a pattern by a specifically implemented activity template (cf. Fig. 6(2)) is applied in the context of the Parallel Pattern I as well (cf. Fig. 6). However, there is another case to be considered by application developers. In certain situations, it needs to be evaluated, whether or not the activities shall be executed concurrently. As existing mobile process engines [9] do not allow for a concurrent execution, a specifically implemented
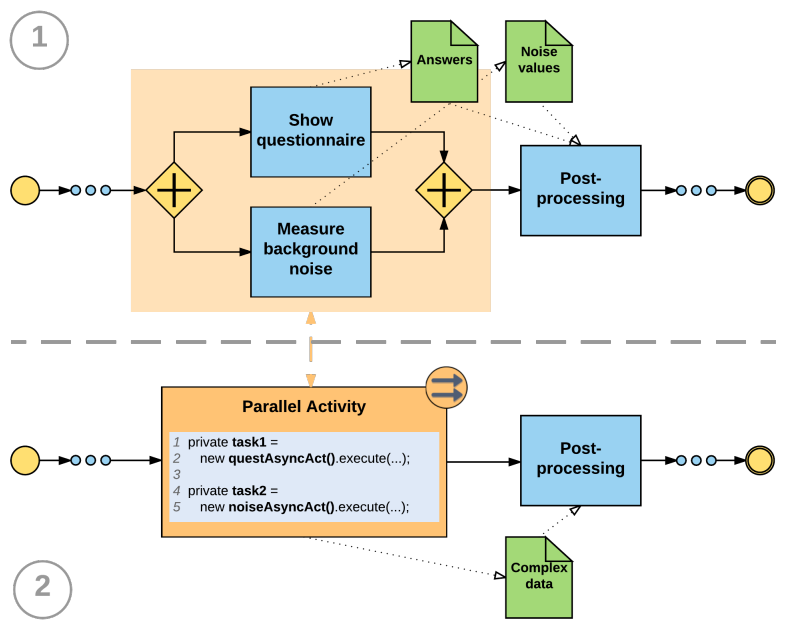

Figure 6: Parallel Pattern I 


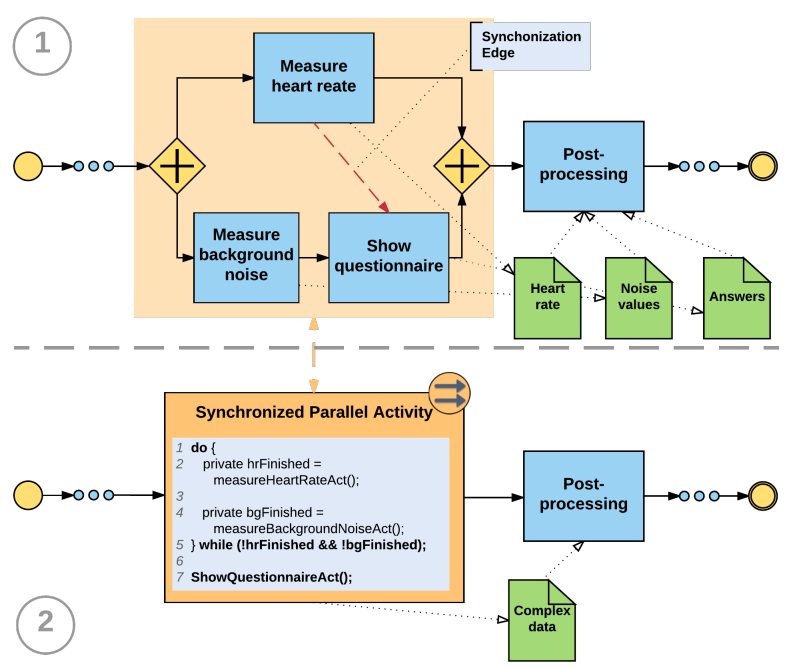

Figure 7: Parallel Pattern II

activity template is required.

Note that the approach of replacing a sub-process pattern by a specifically implemented activity template constitutes the basis for the next two sub-process patterns as well. Therefore, only new aspects are discussed.

\section{Parallel Pattern II}

This sub-process pattern differs in one respect compared to the latter pattern. Two of the parallel activities are associated with an entailment constraint, which is visualized by a synchronization edge (cf. Fig. 7). The latter expresses that the target activity may only be started when the source activity as well as all other preceding activities will have been finished. In the example of Fig. 7, two sensor activities shall measure data in parallel, whereas a questionnaire asking the patient about aspects of the exercise shall only be provided after completing both measurements. In practice, the realization of the Parallel Pattern II is challenging on one hand. On the other, we learned that this pattern is useful and frequently demanded by therapists. Consequently, we consider it as an independent sub-process pattern.

\section{Loop Pattern}

The Loop Pattern is shown in Fig. 8. It covers the case that activities may be executed multiple times. Again, this sub-process pattern is practically challenging as well. For example, in cases where sensor activities shall be executed multiple times, a specifically implemented activity template is frequently used. Opposed to that, no specifically implemented activity template is used if human activities shall be performed multiple times.

\section{Discussion}

This section discusses how our mobile processes address the requirements presented in Table I. Due to space limitations, we focus on one particular aspect of each of the three requirement categories. First, for the homework category, mobile processes enable the therapist to reuse homework more easily. For example, a homework containing a breathing exercise in the context of mental disorders can be easily customized to reuse it for a chronical back pain intervention. Therefore, only the context for the chronical back pain intervention must be changed. Note that the customization and reuse approach do not harm the outcome of therapeutic interventions. Second, for the context category, mobile processes enable the use of advanced notifications. As notifications themselves may be represented by a mobile process fragment, sophisticated procedures become possible. For example, before a notification is provided, the smart mobile device measures the moment-to-moment heart rate. Third, for the smart mobile device category, mobile processes enable the flexible use of sensors as their use can be represented in terms of activity templates. For example, if a sensor measurement shall take place at the beginning of an exercise or at its end, can be only determined by placing the sensor activity to the desired position.

Furthermore, three additional aspects require a brief discussion. First, regarding the pursued objectives (cf. Fig. 1), we believe that the involvement of researchers in therapeutic interventions is promising. Mobile processes, in turn, support this objective as the entire therapy as well as each single activity gets a digital footprint. The newly gathered data can then be automatically evaluated and new medical insights potentially be revealed in order to enhance existing therapeutic interventions. Second, the mobile processes enable us to assist an application developer when (1) developing new activity templates and (2) evaluating the identified sub-process patterns. Third, legal and privacy aspects are currently not considered. However, many concepts exist
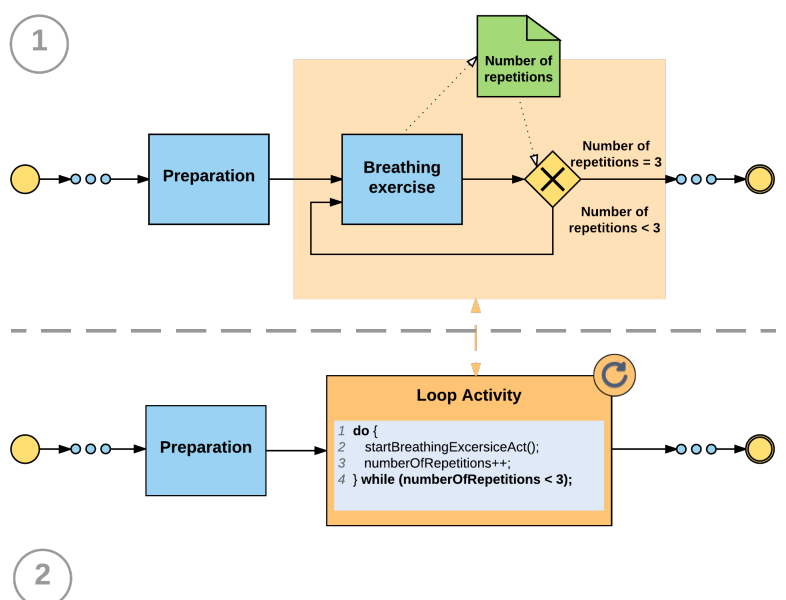

Figure 8: Loop Pattern 
in this context, which are currently evaluated to identify whether they can be integrated with our approach.

\section{PROOF-OF-CONCEPT PROTOTYPE}

We realized a proof-of-concept prototype called Albatros. Note that the prototype is based on a sophisticated architecure that cannot be presented in more detail due to space limitations. Inspired by the insights we gained in the QuestionSys project [10], our modeling component has been developed. However, the two projects Albatros and QuestionSys differ significantly with respect to their use of mobile processes. In the Albatros project, we focus on run time adaptations of mobile process instances (e.g., adding an ad-hoc activity) based on monitoring data, whereas in the QuestionSys project, the monitoring of mobile process instances is not required. Currently, the prototype of the Albatros project consists of an HTML5 application and a mobile application running on Android. The HTML5 application provides the modeling component as well as the component for monitoring and managing therapeutic interventions by therapists. The Android application, in turn, integrates the mobile process engine, which we developed in the QuestionSys project [8], to enact process models on smart mobile devices.

Figs. 9 and 10 show selected features of the prototype. Fig. 9 presents the view provided to therapists for managing entire patient cases. More precisely, therapeutic interven-

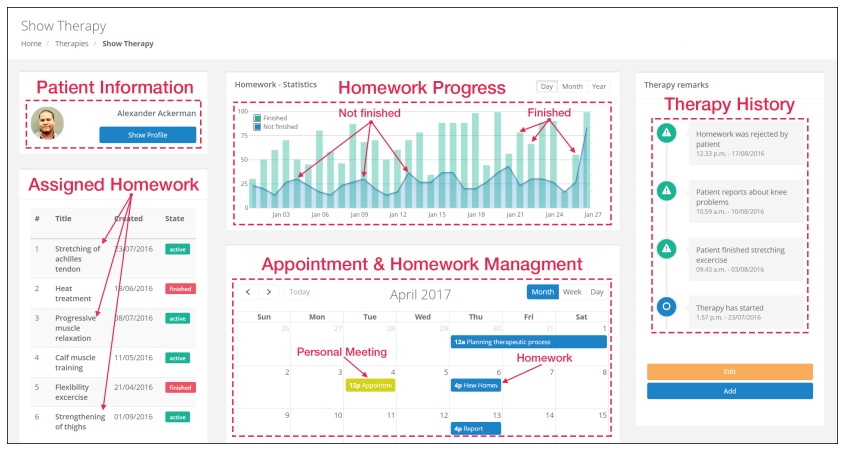

Figure 9: Therapy Details

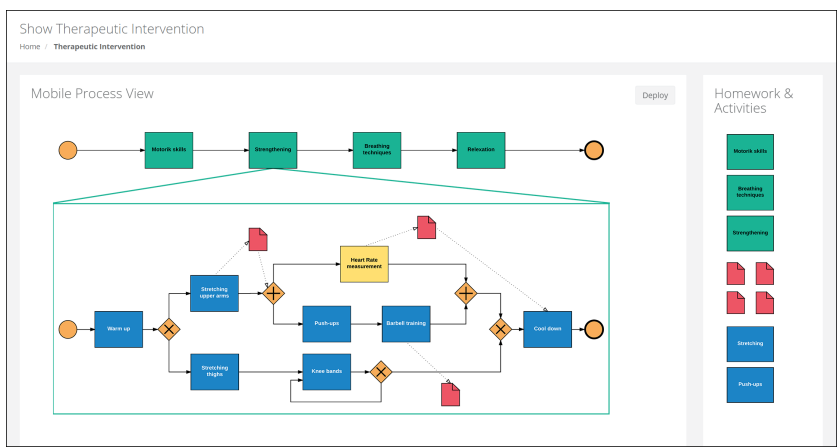

Figure 10: Mobile Process tions, personal appointments, and anamnesis are managed using this view. In turn, Fig. 10 presents the view of a therapeutic intervention before deploying it to the smart mobile device. Note that the deployed mobile process may be visually displayed.

\section{RELATED WORK}

Three categories of related work are relevant in the context of this paper.

Approaches dealing with mobile therapies. In [11], several studies are presented that use mobile phones as a motivating factor for teenagers to record their mental health symptoms. The recordings, in turn, are discussed in the clinic with the therapists. In [12], various approaches using smartphones in the context of personal healthcare are presented. Interestingly, these approaches confirm that contemporary solutions less focus on mobile therapies, although beneficial use of interventions is emphasized by most of them. In line with [12], [3] underlines the benefits of mobile technology regarding the efficacy of psychotherapy. None of these approaches presents technical solutions for therapeutic interventions as provided by us.

[13] presents three clinical studies in which mobile technology was used for mobile interventions. For each study, a specifically tailored mobile application is realized. Although [13] reveals that mobile interventions were useful in the context of these case studies, no generic technical solution is presented.

Approaches dealing with model-driven guidance in the context of mobile healthcare users. Recently, approaches have been introduced that use model-driven techniques. The QuestionSys approach presented in [8], [14], [15], for example, allows realizing mobile data collection applications by healthcare experts (i.e., without any support by IT experts). Another model-driven approach is presented in [16]; it presents a framework that automatically creates healthcare plans based on a model-driven concept. The plans, in turn, are deployed to smartphones. In [17], a modeldriven approach in connection with Body Area Networks (BANs) is presented. It considers various BAN scenarios involving medical expert as well as patients more properly.

Approaches dealing with homework in the context of psychotherapy. Numerous related works deal with homework in the context of psychotherapy [11], [18], [19]. However, they mainly focus on user studies instead of technical solutions. In turn, all of these studies revealed that homework should be a fundamental pillar of modern psychotherapies.

Altogether, related work has identified homework as being fundamental in the context of many therapies [6]. The development of a generic technical solution, which supports homework in particular and therapeutic interventions in general, is currently less considered. 


\section{Summary AND OUTLOOK}

This paper presented a concept based on mobile processes, which provide the basis for a protocol as well as a technical solution enabling therapists to provide remote therapeutic interventions to their patients with the help of smart mobile devices. We presented fundamental requirements elaborated in real-world projects in order to capture the practical demands of therapists on one hand and the expectations of patients on the other. As a major goal, therapeutic interventions shall be flexibly applied to patients by the use of their smart mobile devices. To flexibly cope with the demands of therapists and to enable personalized treatment, the support of mobile processes is a decisive step towards a resilient technical solution. As shown, mobile processes are built up on different conceptual levels, which are the fundamental pillar to automatically transform therapeutic interventions to executable mobile processes. Furthermore, mobile processes support application developers in realizing new activity templates and sub-processes for therapeutic interventions. Especially, in the context of therapeutic interventions, the support of application developers is of utmost importance due to non-foreseeable scenarios. We further sketched our proof-of-concept prototype, which has already proven its applicability in practice. Overall, the presented approach is promising to properly support remote therapeutic interventions on smart mobile devices. In future work, we focus on two issues. First, the modeling component is to be evaluated through an experiment. Second, the mobile application is implemented on iOS as well as on Windows Phone. Altogether, the support of therapeutic interventions on smart mobile devices constitutes an important step towards more effective therapies.

\section{REFERENCES}

[1] A. Aguilera and F. Muench, "There's an app for that: Information technology applications for cognitive behavioral practitioners," The Behavior therapist/AABT, vol. 35, no. 4, p. $65,2012$.

[2] S. Watts et al., "Cbt for depression: a pilot ret comparing mobile phone vs. computer," BMC psychiatry, vol. 13, no. 1, p. 49, 2013.

[3] O. Lindhiem, C. Bennett, D. Rosen, and J. Silk, "Mobile technology boosts the effectiveness of psychotherapy and behavioral interventions: A meta-analysis," Behavior modification, vol. 39, no. 6, pp. 785-804, 2015.

[4] B. Clough and L. Casey, "Therapy on the move: The development of a therapeutic smartphone application," Int'l Journal of Cyber Behavior, Psychology and Learning, vol. 5, no. 1, pp. 33-41, 2015.

[5] T. Probst et al., "Feedback on patient progress and clinical support tools for therapists: Improved outcome for patients at risk of treatment failure in psychosomatic in-patient therapy under the conditions of routine practice," Journal of Psychosomatic Research, vol. 75, no. 3, pp. 255-261, 2013.
[6] _ - "Providing patient progress feedback and clinical support tools to therapists: Is the therapeutic process of patients on-track to recovery enhanced in psychosomatic in-patient therapy under the conditions of routine practice?" Journal of psychosomatic research, vol. 76, no. 6, pp. 477-484, 2014.

[7] M. Schickler et al., "Using Mobile Serious Games in the Context of Chronic Disorders - A Mobile Game Concept for the Treatment of Tinnitus," in 29th IEEE Int'l Symp on Computer-Based Medical Systems, 2016, pp. 343-348.

[8] J. Schobel et al., "A Mobile Service Engine Enabling Complex Data Collection Applications," in 14th Int'l Conf on Service Oriented Computing, ser. LNCS, 2016, pp. 626-633.

[9] J. Schobel, R. Pryss, M. Schickler, and M. Reichert, "A lightweight process engine for enabling advanced mobile applications," in 24th Int'l Conf. on Cooperative Information Systems. Springer, 2016, pp. 552-569.

[10] — "A Configurator Component for End-User Defined Mobile Data Collection Processes," in Proc 14th Int'l Conf on Service Oriented Computing, October 2016.

[11] D. J. Cox, D. A. Tisdelle, and J. Culbert, "Increasing adherence to behavioral homework assignments," Journal of behavioral medicine, vol. 11, no. 5, pp. 519-522, 1988.

[12] K. Wac, "Smartphone as a personal, pervasive health informatics services platform: literature review," arXiv preprint arXiv:1310.7965, 2013.

[13] C. Depp et al., "Mobile interventions for severe mental illness: design and preliminary data from three approaches," The Journal of nervous and mental disease, vol. 198, no. 10, p. 715, 2010.

[14] J. Schobel et al., "Towards Process-Driven Mobile Data Collection Applications: Requirements, Challenges, Lessons Learned," in 10th Int'l Conf on Web Information Systems and Technologies, 2014, pp. 371-382.

[15] _ _ "End-User Programming of Mobile Services: Empowering Domain Experts to Implement Mobile Data Collection Applications," in 5th IEEE Int'l Conf on Mobile Services, 2016, pp. 1-8.

[16] A. Khambati, J. Grundy, J. Warren, and J. Hosking, "Modeldriven development of mobile personal health care applications," in Proc of the 23rd IEEE/ACM Int'l Conf on Automated Software Engineering. IEEE Computer Society, 2008, pp. 467-470.

[17] V. Jones, A. Rensink, and E. Brinksma, "Modelling mobile health systems: an application of augmented MDA for the extended healthcare enterprise," in 9th IEEE Int'l Conf on Enterprise Computing). IEEE, 2005, pp. 58-69.

[18] N. Kazantzis, C. Whittington, and F. Dattilio, "Meta-analysis of homework effects in cognitive and behavioral therapy: A replication and extension," Clinical Psychology: Science and Practice, vol. 17, no. 2, pp. 144-156, 2010.

[19] F. Leichsenring et al., "The empirical status of psychodynamic psychotherapy-an update: Bambi's alive and kicking," Psychotherapy and psychosomatics, vol. 84, no. 3, pp. 129 148, 2015. 